\title{
Natural Coordinate System in Curved Space-time
}

\author{
Ying-Qiu $\mathrm{Gu}^{*}$ \\ School of Mathematical Science, Fudan University, Shanghai 200433, China
}

(Dated: 28th October 2017)

\begin{abstract}
In this paper we establish a generally and globally valid coordinate system in curved space-time with the simultaneous hypersurface orthogonal to the time coordinate. The time coordinate can be preseted according to practical evolving process and keep synchronous with the evolution of the realistic world. In this coordinate system, it is convenient to express the physical laws and to calculate physical variables with clear geometrical meaning. We call it "natural coordinate system". The constructing method for the natural coordinate system is concretely provided, and its physical and geometrical meanings are discussed in detail. In NCS we make classical approximation of spinor equation to get Newtonian mechanics, and then make weak field approximation of Einstein's equation and low speed approximation of particles moving in the space-time. From the analysis and examples we find it is a nice coordinate system to describe the realistic curved space-time, and is helpful to understand the nature of space-time.
\end{abstract}

Keywords: coordinate system, orthogonalization, simultaneity, canonical metric

PACS numbers: 04.20-q, 04.20.Dw, 04.20.Jb, 04.25-g

\section{INTRODUCTION}

The selection of coordinate system in general relativity is important to conveniently express and solve physical equations. In a suitable coordinate system, the physical equations have simple and concise forms and definite physical meanings. In general relativity, the special coordinate systems we usually used for theoretical discussion are Gaussian normal coordinate system and harmonic coordinate system[1]. However, the Gaussian normal coordinate system only exists locally. The geometrical meaning of harmonic coordinate system is unclear. Other coordinate systems, such as Weyl-Lewis- Papapetrou one[2], all depend on special structure of space-time.

A more important problem is that, the realistic space-time is an evolving Lorentz manifold which has one and only one simultaneous hypersurface $f\left(x^{\mu}\right)=C[3,4]$. The hypersurface forms

*Electronic address: yqgu@fudan.edu.cn 
the realistic space and evolves from the present state to the next one. Since the evolution of the world has probabilistic characteristic of quantum mechanics, it is obviously non-unique. In principle, it is most natural and reasonable to describe physical laws in the coordinate system with simultaneity $t=t_{k}$ coincident with the evolving hypersurfaces. Besides, it is perfect if the time coordinate is orthogonal to this simultaneous hypersurface similarly to the case in Gaussian normal coordinate system, because only in such coordinate system, we can conveniently discuss the Hamiltonian formalism of dynamics and calculate Nöther's charges. We call the coordinate system with such two features "natural coordinate system (NCS)". The purpose of this paper is to prove the existence of NCS and look for a general method to establish such NCS, and then clarify its physical meanings.

To get canonical quantification of gravity[5,6] or numerical simulation of Einstein' field equation[7, 8], we also need to define a simultaneous Cauchy hypersurface. The current treatment is the ADM decomposition of space-time[5]. In ADM decomposition, the metric is reduced into time plus space with a shift vector $N^{j}$,

$$
d s^{2}=\mathcal{N}^{2} d t^{2}+\widetilde{g}_{k l}\left(d x^{k}+N^{k} d t\right)\left(d x^{l}+N^{l} d t\right)
$$

where the repeated Latin indices means summation of spatial indices. The Ricci tensor and scalar curvature can be expressed in simple and reduced from. If we can set the shift vector $N^{j}=0$ and clarify the specific geometrical meaning of space and time, the formalism will be even simpler.

\section{CONSTRUCTION OF NATURAL COORDINATE SYSTEM}

At first, we discuss the problem of orthogonalization. We use the Greek characters such as $\mu, \nu$ to stand for the 4 -d coordinate indices, and Latin characters such as $k, l$ for 3 -d indices. Assume the metric of the space-time is given by

$$
d s^{2}=\widetilde{g}_{t t} d t^{2}+2 A_{k} d t d x^{k}+\widetilde{g}_{k l} d x^{k} d x^{l}, \quad A_{k} \equiv \widetilde{g}_{0 k}
$$

we have the following conclusion for orthogonalization.

Theorem 1. Keeping the time coordinate $t$ not transformed, for (2.1) there exists a class of regular spatial coordinate transformation

$$
x^{k}=x^{k}\left(t, y^{l}\right), \quad d x^{k}=\frac{\partial x^{k}}{\partial t} d t+\frac{\partial x^{k}}{\partial y^{l}} d y^{l},
$$

such that ' $d t$ ' is orthogonal to ' $d y^{k}$ ', i.e., in the new coordinate system we have

$$
d s^{2}=g_{t t} d t^{2}-g_{k l} d y^{k} d y^{l} .
$$


Proof. Substituting (2.2) into (2.1), we get

$$
d s^{2}=g_{t t} d t^{2}+2 B_{k} d t d y^{k}-g_{k l} d y^{k} d y^{l} .
$$

in which

$$
B_{k} \equiv\left(\widetilde{g}_{n l} \frac{\partial x^{l}}{\partial t}+A_{n}\right) \frac{\partial x^{n}}{\partial y^{k}}
$$

Since the transformation (2.2) should be invertible, the Jacobian matrix $\frac{\partial x^{l}}{\partial y^{k}}$ also is invertible. Then $B_{k}=0$ is equivalent to

$$
\partial_{t} x^{k}=-\bar{g}^{k l} A_{l}=-\bar{g}^{k l} \widetilde{g}_{0 l}
$$

where $\bar{g}^{k l}$ is the inversion of spatial metric, i.e., $\widetilde{g}_{k l} \bar{g}^{l n}=\delta_{k}^{n}$.

Since the time coordinate $t$ is a fixed independent coordinate, (2.6) becomes a first order ordinary differential equation system of $x^{k}$. For the initial value problem we have unique solution

$$
x^{k}=f^{k}\left(t, X^{l}\right)
$$

in which $X^{k}$ is integral constants determined by initial values. Making an arbitrary invertible and differentiable coordinate transformation

$$
X^{k}=F^{k}\left(y^{l}\right),
$$

and substituting it into (2.7) we get (2.2). The proof is finished.

Obviously, if $g_{k l}$ in (2.3) is independent of $t$, the above procedure of orthogonalization can proceed, such that $d y^{1}$ is orthogonal to the other two coordinates. Since the proof is constructive, it can also be used as method to establish the NCS.

In what follows we examine the problem of simultaneity. In [3, 4], we have proved that, in the realistic space-time there exists one and only one simultaneous hypersurface $f\left(x^{\mu}\right)=C$, which is an objective existence independent of coordinate system. According to its physical meanings, the hypersurface should have the following two features: F1. Assuming $d x^{0}$ is time-like, by the unidirection of time we have $\partial_{0} f>0$ under a suitable function transformation of $f\left(x^{\mu}\right)$. F2. $f\left(x^{\mu}\right)$ has higher smoothness than $C^{1}$, this is because all physical dynamics consists of first or second order differential equation system. We take F1 and F2 as basic assumptions for the simultaneous hypersurface. Redefine the time coordinate

$$
t=f\left(x^{\mu}\right),
$$


then the simultaneity of the real world becomes $t \equiv t_{k}$, which defines the global realistic simultaneity. We call it the "cosmic time"[9]. Clearly, if we make orthogonalization based on this cosmic time, we get the NCS (2.3) with special significances. We call the space orthogonal to the cosmic time as "cosmic space". Obviously, the coordinate system for cosmic time and cosmic space can be determined to the following arbitrary regular transformation

$$
t^{\prime}=T(t),\left(\partial_{t} T>0\right), \quad y^{\prime k}=Y^{k}\left(y^{l}\right) .
$$

However, the time element $d \tau=\sqrt{g_{00}} d t$ and the spatial volume element $d V=\sqrt{\bar{g}} d^{3} y$ are objective quantities independent of transformation (2.10), where $\bar{g}=\operatorname{det}\left(g_{k l}\right)$. Then we proved,

Theorem 2. In the realistic world we have a unique global cosmic time $t$ and a unique cosmic space orthogonal to this time. The objective time is calculated by $d \tau=\sqrt{g_{00}} d t$, and the objective volume of the space is calculated by $d V=\sqrt{\bar{g}} d^{3} y$. They are independent of coordinate system.

In contrast with AMD decomposition, in NCS the shift vector is removed and the time and space have endowed with concrete physical meanings. The NCS (2.3) looks like Gaussian normal coordinate system, but their physical meanings are different: M1. NCS is always globally valid, and $g_{t t}$ represents gravity which cannot be merged into coordinate $t$ in the case $\partial_{k} g_{t t} \not \equiv 0$. M2. The construction is different. NCS is independent of geodesic of concrete particles. M3. The time $t$ in NCS corresponds to realistic cosmic time, which forms a global standard of time for all particles. Since the construction of NCS does not need any special conditions, we can directly express physical laws in this coordinate system without any declaration.

Now we concretely construct a NCS from a non-diagonal Kerr-type metric[2]. More generally, the metric takes the following form in the coordinate system $(t, r, \theta, \phi)$,

$$
\widetilde{g}_{\mu \nu}=\left(\begin{array}{cccc}
u^{2} & 0 & 0 & u w \\
0 & -a & 0 & 0 \\
0 & 0 & -b & 0 \\
u w & 0 & 0 & w^{2}-v
\end{array}\right), \quad \widetilde{g}^{\mu \nu}=\left(\begin{array}{cccc}
\frac{-w^{2}+v}{u^{2} v} & 0 & 0 & \frac{w}{u v} \\
0 & -a^{-1} & 0 & 0 \\
0 & 0 & -b^{-1} & 0 \\
\frac{w}{u v} & 0 & 0 & -v^{-1}
\end{array}\right) \text {. }
$$

where $u, v, w, a, b$ are smooth functions of $(r, \theta)$, but independent of $(t, \phi)$. However we should pay attention to that

$$
\widetilde{g}_{k l}=\left(\begin{array}{ccc}
-a & 0 & 0 \\
0 & -b & 0 \\
0 & 0 & w^{2}-v
\end{array}\right), \quad \bar{g}^{k l}=\left(\begin{array}{ccc}
-a^{-1} & 0 & 0 \\
0 & -b^{-1} & 0 \\
0 & 0 & -\left(v-w^{2}\right)^{-1}
\end{array}\right) .
$$


Assuming that $t$ is the cosmic time, then by (2.6) we have

$$
\partial_{t} r=0, \quad \partial_{t} \theta=0, \quad \partial_{t} \phi=-\bar{g}^{\phi \phi} \widetilde{g}_{t \phi}=\left(v-w^{2}\right)^{-1} u w .
$$

The simplest solution is given by

$$
r=r^{\prime}, \quad \theta=\theta^{\prime}, \quad \phi=\left(v-w^{2}\right)^{-1} u w t+\phi^{\prime} .
$$

It is easy to check, the metric (2.11) is converted into the standard one (2.3) in new coordinate system $\left(t, r^{\prime}, \theta^{\prime}, \phi^{\prime}\right)$. The coordinate $\phi^{\prime}$ becomes an evolving one. The clock keeping static to this coordinate system goes in uniform speed. This is somewhat similar to the Mach's principle[10, 11].

\section{APPLICATIONS}

\section{A. Classical Approximation of Spinor Equation}

To discuss the Hamiltonian formalism of Dirac equation in curved space-time we need the cosmic time coordinate with clear physical meaning and orthogonal to space. To calculate the Nöther charges of a spinor such as energy-momentum and velocity, we need to do integration on the realistic cosmic space. Some works have been done in Gaussian normal coordinate system[12]. Now we define some classical concepts in NCS to show the advantages.

Assume the element of the space-time satisfies

$$
d \mathbf{x}=\widetilde{\gamma}_{\mu} d x^{\mu}=\gamma_{a} \delta X^{a}, \quad \widetilde{\gamma}_{\mu}=l_{\mu}^{a} \gamma_{a}, \quad \widetilde{\gamma}^{\mu}=h^{\mu}{ }_{a} \gamma^{a}
$$

in which the tetrad $\gamma_{\alpha}$ and $\widetilde{\gamma}_{\mu}$ satisfy the following $C \ell_{1,3}$ Clifford algebra,

$$
\gamma_{a} \gamma_{b}+\gamma_{b} \gamma_{a}=2 \eta_{a b}, \quad \widetilde{\gamma}_{\mu} \widetilde{\gamma}_{\nu}+\widetilde{\gamma}_{\nu} \widetilde{\gamma}_{\mu}=2 g_{\mu \nu}
$$

In NCS with metric $\operatorname{diag}\left(g_{00},-\bar{g}_{j k}\right)$, we have

$$
l_{0}^{0}=\sqrt{g_{00}}, \quad h_{0}^{0}=\sqrt{g^{00}}, \quad h_{k}^{0}=l_{0}^{k}=0 .
$$

Then we get spinor connection as[12]

$$
\Upsilon_{\mu}=\frac{1}{2}\left(l_{0}^{0} \partial_{t} h_{0}^{0}+\partial_{t} \ln \sqrt{g}, \vec{l}_{k} \cdot \partial_{j} \vec{h}^{j}+\partial_{k} \ln \sqrt{g}\right) .
$$

In NCS, to lift and lower the index of a vector means $\Upsilon^{0}=g^{00} \Upsilon_{0}, \Upsilon^{k}=-\bar{g}^{k l} \Upsilon_{l}$.

We consider Dirac equation with electromagnetic potential $e A^{\mu}$. Its Hamiltonian formalism is given by

$$
\alpha^{0} i\left(\partial_{t}+\Upsilon_{t}\right) \phi=\mathbf{H} \phi
$$


where the Hamiltonian is defined by

$$
\mathbf{H}=-\alpha^{k} \cdot\left[i\left(\partial_{k}+\Upsilon_{k}\right)-e A_{k}\right]+e \alpha^{0} A_{0}+m \gamma_{0}, \quad\left(\alpha^{\mu} \equiv \gamma_{0} \widetilde{\gamma}^{\mu}\right)
$$

Similarly to the case in flat space-time, we define the coordinate $X^{k}$ and speed $v^{k}$ of the spinor as follows[12, 13],

$$
X^{k}(t) \equiv \int_{S^{3}} x^{k} q^{0} \sqrt{g} d^{3} x, \quad v^{k} \equiv \frac{d}{d t} X^{k}
$$

where $S^{3}$ stands for the total simultaneous hypersurface, i.e., the cosmic space, $q^{\mu}$ is the current vector $q^{\mu}=\phi^{+} \alpha^{\mu} \phi$. By the definition (3.7) and the current conservation law $q_{; \mu}^{\mu}=0$, it is easy to check

$$
v^{k}=\int_{S^{3}} x^{k} \partial_{t}\left(q^{0} \sqrt{g}\right) d^{3} x=\int_{S^{3}} x^{k} q_{; t}^{0} \sqrt{g} d^{3} x=-\int_{S^{3}} x^{k} q_{; l}^{l} \sqrt{g} d^{3} x=\int_{S^{3}} q^{k} \sqrt{g} d^{3} x .
$$

With the normalizing condition $\int_{S^{3}} q^{0} \sqrt{g} d^{3} x=1$, we have the point-particle model,

$$
q^{\mu} \rightarrow u^{\mu} \sqrt{g_{00}-\bar{g}_{k l} v^{k} v^{l}} \delta^{3}(\vec{x}-\vec{X}), \quad u^{\mu} \equiv \frac{d X^{\mu}}{d \tau}=(1, \vec{v}) / \sqrt{g_{00}-\bar{g}_{k l} v^{k} v^{l}},
$$

where the Dirac- $\delta$ means $\int_{S^{3}} \delta^{3}(\vec{x}-\vec{X}) \sqrt{\bar{g}} d^{3} x=1$ and $\tau$ is the proper time of the particle $d \tau=$ $\sqrt{g_{00}-\bar{g}_{k l} v^{k} v^{l}} d t$.

Clearly, the particle static to NCS has largest proper time, i.e., its time goes fastest. Since the time of any particle should go at a finite speed, which certainly have a least upper bound everywhere, this requirement also discloses the existence of a special coordinate system in Nature. By the definition of NCS, we find that the cosmic time and cosmic space are quite near the concepts of Galilean and Newtonian absolute space-time. The classical concept of space-time cannot be completely cast away.

Define the 4-dimensional momentum of the spinor by

$$
p^{\mu} \equiv \Re \int_{S^{3}} \phi^{+} \alpha^{0} \hat{p}^{\mu} \phi \sqrt{g} d^{3} x=\Re \int_{S^{3}} \phi^{+} \hat{p}^{\mu} \phi \sqrt{\bar{g}} d^{3} x, \quad \hat{p}^{\mu} \equiv i\left(\partial^{\mu}+\Upsilon^{\mu}\right)-e A^{\mu} .
$$

For a spinor at energy eigenstate, we have the classical approximation $p^{\mu}=m u^{\mu}$, where $m$ defines the classical inertial mass of the spinor. The classical approximation of energy-momentum tensor $T^{\mu \nu}$ for a free spinor is given by

$$
T^{\mu \nu} \rightarrow\left(m u^{\mu} u^{\nu}+w g^{\mu \nu}\right) \sqrt{1-g^{00} \bar{g}_{k l} v^{k} v^{l}} \delta^{3}(\vec{x}-\vec{X}),
$$

where $w$ is a constant to representing self-potential, and $w=0$ corresponds to linear spinor. By $T_{; \nu}^{\mu \nu}=0$ we get

$$
\partial_{\nu}\left(T^{\mu \nu} \sqrt{g}\right)+\Gamma_{\alpha \beta}^{\mu} T^{\alpha \beta} \sqrt{g}=0 .
$$


The integral form is given by

$$
\frac{d}{d t} \int_{S^{3}} T^{\mu 0} \sqrt{g} d^{3} x+\int_{S^{3}} \Gamma_{\alpha \beta}^{\mu} T^{\alpha \beta} \sqrt{g} d^{3} x=0 .
$$

Substituting (3.11) into (3.13) and noticing $g=g_{00} \bar{g}$, we get the Newton's second law for a free spinor

$$
\frac{d}{d t}\left(m u^{\mu}+w g^{\mu 0} \sqrt{g_{00}-\bar{g}_{k l} v^{k} v^{l}}\right)+\Gamma_{\alpha \beta}^{\mu}\left(m u^{\alpha} u^{\beta}+w g^{\alpha \beta}\right) \sqrt{g_{00}-\bar{g}_{k l} v^{k} v^{l}}=0 .
$$

In the case of linear spinor we have $w=0$, and then (3.14) becomes the geodesic equation. From the above calculation we find that, some Nöther's charges of fields can be clearly defined and computed only in NCS. So NCS set up a natural connection between quantum mechanics and classical one.

\section{B. Linearization of Einstein's Field Equation}

In the case of weak gravity and low speeds of particles, we make linearization of Einstein's field equation and get a set of wave equation in harmonic coordinate system with the de Donder coordinate condition $\Gamma^{\mu}=0$, where

$$
\Gamma^{\mu} \equiv g^{\alpha \beta} \Gamma_{\alpha \beta}^{\mu}=-\frac{1}{\sqrt{g}} \partial_{\nu}\left(\sqrt{g} g^{\mu \nu}\right) .
$$

In this case the metric is near the Minkowski metric

$$
\eta_{\mu \nu}=\eta^{\mu \nu}=\operatorname{diag}(1,-1,-1,-1)
$$

For weak-field approximation, we have the linearization for the metric[1]

$$
\begin{aligned}
g_{\mu \nu} & \equiv \eta_{\mu \nu}+h_{\mu \nu}, & g^{\mu \nu} \doteq \eta^{\mu \nu}-h^{\mu \nu}, \\
h^{\mu \nu} & =\eta^{\mu \alpha} \eta^{\nu \beta} h_{\alpha \beta}, & h=h^{\mu}{ }_{\mu}=\eta^{\mu \nu} h_{\mu \nu}, \\
g & \doteq 1+h, & \sqrt{g} \doteq 1+\frac{1}{2} h .
\end{aligned}
$$

In what follows, we directly use $=$ to replace $\doteq$ for convenience. By straightforward calculation, we get the linearization for other parameters

$$
\begin{aligned}
\Gamma_{\alpha \beta}^{\mu} & =\frac{1}{2} \eta^{\mu \nu}\left(\partial_{\alpha} h_{\nu \beta}+\partial_{\beta} h_{\alpha \nu}-\partial_{\nu} h_{\alpha \beta}\right), \\
\Gamma^{\mu} & =\partial_{\nu} \chi^{\mu \nu}, \quad \chi^{\mu \nu} \equiv h^{\mu \nu}-\frac{1}{2} \eta^{\mu \nu} h, \\
R_{\mu \nu} & =\frac{1}{2} \partial_{\alpha} \partial^{\alpha} h_{\mu \nu}-\frac{1}{2}\left(\eta_{\mu \alpha} \partial_{\nu} \Gamma^{\alpha}+\eta_{\nu \alpha} \partial_{\mu} \Gamma^{\alpha}\right), \\
R & =\frac{1}{2} \partial_{\alpha} \partial^{\alpha} h-\partial_{\alpha} \Gamma^{\alpha}, \\
G^{\mu \nu} & =\frac{1}{2}\left(\partial_{\alpha} \partial^{\alpha} \chi^{\mu \nu}-\partial^{\mu} \Gamma^{\nu}-\partial^{\nu} \Gamma^{\mu}+\eta^{\mu \nu} \partial_{\alpha} \Gamma^{\alpha}\right),
\end{aligned}
$$


in which $\partial_{\alpha} \partial^{\alpha}=\partial_{t}^{2}-\Delta$ is the d'Alembert operator. By (3.24) we find the Bianchi identity $\partial_{\mu} G^{\mu \nu}=0$ holds.

In NCS the time coordinate is fixed, so we have not coordinate condition $\Gamma^{\mu}=0$ in general. However, by $G^{0 \mu}$ we find $\Gamma^{\mu}$ can be easily solved independently in NCS. Explicitly we have

$$
\begin{aligned}
\Gamma^{0} & =\partial_{t} \Psi, \quad \Gamma^{k}=\frac{1}{2} \partial_{k} h+\partial_{n} h_{k n}, \\
\Psi & \equiv \chi^{00}=\frac{1}{2}\left(h_{00}+h_{11}+h_{22}+h_{33}\right),
\end{aligned}
$$

where the repeated Latin indices means summation of spatial indices. Then by $G^{0 \mu}$ we get independent equations for $\left(\Psi, \Gamma^{k}\right)$ as follows

$$
\begin{aligned}
G^{00} & =-\frac{1}{2} \Delta \Psi=\kappa T^{00}, \\
G^{0 k} & =-\frac{1}{2} \partial_{t}\left(\Gamma^{k}-\partial_{k} \Psi\right)=\kappa T^{0 k} .
\end{aligned}
$$

By the above equations we can determine the intermediate variables $\Gamma^{\mu}$. Substituting it into (3.24) we get usual wave equations for $h_{k l}$.

From the procedure we find the coordinate condition is determined by dynamics as well as the initial and boundary values. This is natural, because the simultaneous hypersurface is evolving itself.

Now we examine the classical particles moving in the weak gravity. Under low speed assumption we omit $O\left(v^{2}\right)$. Noticing $v^{k} \equiv \frac{d}{d t} X^{k}=\sqrt{1+h_{00}} \frac{d}{d \tau} X^{k}$, the geodesic equation becomes

$$
\frac{d}{d t} v^{k}=-\partial_{k} \Phi+v^{k} \partial_{t} \Phi+v^{n} \partial_{t} h_{k n}
$$

where $\Phi=\frac{1}{2} h_{00}$ is the Newtonian gravitational potential. For many such particles move without collision similarly to stars in a galaxy, which form zero-pressure and inviscid fluid with following energy-momentum tensor

$$
T^{\mu \nu}=\rho U^{\mu} U^{\nu}
$$

where $\rho$ is the comoving mass density of the stars, and $U^{\mu}$ is the 4 -vector speed of the stellar flow. We have energy-momentum conservation law $T_{; \nu}^{\mu \nu}=0$. Expressing it in the form of equations of continuity and motion, we get the dynamical equations for the stars

$$
U^{\mu} \partial_{\mu} \rho+\rho_{s} U_{; \mu}^{\mu}=0, \quad U^{\nu} U_{; \nu}^{\mu}=0 .
$$

Define the stellar speed $\vec{V}$ by

$$
\vec{V} \equiv \frac{1}{U^{0}}\left(U^{1}, U^{2}, U^{3}\right)
$$


which is approximately equivalent to the usual definition of velocity $\frac{d}{d t} \vec{X}$. By line element equation we have

$$
1=\sqrt{g_{\mu \nu} U^{\mu} U^{\nu}}=\left(1+2 \Phi+g_{k l} V^{k} V^{l}\right)^{\frac{1}{2}} U^{0} .
$$

Omitting $O\left(V^{2}\right)$ terms we get the low-speed assumption

$$
U^{0}=1-\Phi
$$

Substituting (3.32) and (3.34) into (3.31) and omitting the high order terms, we get the continuity equation and motion equation for stars

$$
\begin{aligned}
\frac{d}{d t} \rho & =-\rho \nabla \cdot \vec{V}-\rho\left(\partial_{t}+\vec{V} \cdot \nabla\right) \Psi, \\
\frac{d}{d t} V^{k} & =-\partial_{k} \Phi+V^{k} \partial_{t} \Phi+V^{n} \partial_{t} h_{k n},
\end{aligned}
$$

where $\frac{d}{d t}=\partial_{t}+\vec{V} \cdot \nabla \cdot(3.36)$ and (3.29) have the same form. In NCS, the gravitomagnetic effect vanishes. However, this effect exists in other coordinate system. There are many researches on this problem[14-19]. In usual coordinate system (2.1), we have gravitomagnetic force $\vec{V} \times(\nabla \times \vec{A})$ similar to Lorentz force in electromagnetism.

\section{DISCUSSION AND CONCLUSION}

In this paper, we establish a generally and globally valid natural coordinate system in curved space-time. In this coordinate system, the time and space keep synchronous with the evolution of the realistic world, which have special physical and philosophical meanings. In NCS we can discuss Hamiltonian formalism conveniently as done under ADM decomposition. As examples, we simply make classical approximation of spinor equation to get Newtonian mechanics, which clearly shows the relationship between quantum mechanics, classical mechanics and general relativity. By weak field approximation of Einstein's equation and low speed approximation of particles moving in the space-time, we find coordinate condition is included in dynamical equations, and the motion equation of particles has a simple form, in which the gravitomagnetic force vanishes. From the analysis and examples we find the NCS is a nice coordinate system to describe the realistic curved space-time, and is helpful to understand the nature of space-time. 


\section{Acknowledgments}

The author is grateful to his supervisor Prof. Ta-Tsien Li and Prof. Han-Ji Shang for their encouragement.

[1] S. L. Weinberg, Gravitation and Cosmology (Ch7.4,Ch10.4, Ch11.8), Wiley, New York, 1972

[2] J. N. Islam, Rotating fields in general relativity, Cambridge Univ. Press 1985

[3] Y. Q. Gu, Some Subtle Concepts in Fundamental Physics, Physics Essays, Vol.30, 356-363(2017)

[4] Y. Q. Gu, Some Paradoxes in Special Relativity and the Resolutions, Adv. Appl. Cliff. Alg. 21(1), 103-119(2011)

[5] R. Arnowitt, S. Deser and C. W. Misner, The Dynamics of General Relativity, in: "Gravitation: An Intro- duction In Current research",L. Witten (ed.), Wiley, New york, 1962. arXiv:gr-qc/0405109

[6] A. Okolow, ADM-like Hamiltonian formulation of gravity in the teleparallel geometry, Phys. Rev., 124 (3) :925-935, 1996

[7] H Uchida, M Shibata, T Yoshida, Y Sekiguchi, H Umeda, Gravitational Collapse of Rotating Supermassive Stars including Nuclear Burning Effects, Phys. Rev. D 96, 083016 (2017)

[8] W. Baumgarte, S. L. Shapiro, On the Numerical Integration of Einstein's Field Equations Thomas, Phys.Rev. D59 024007 (1999)

[9] Y. Q. Gu, The Quaternion Structure of Space-time and Arrow of Time, J. Mod. Phys. V3, 570$580(2012)$.

[10] C. Rovelli, Quantum Gravity (Ch2.4), Cambridge Univ. Press, Cambridge, England, 2004

[11] CH Brans, Mach's Principle and a Relativistic Theory of Gravitation, Phys. Rev., 124 (3) :925-935, 1996

[12] Y. Q. Gu, The Simplification of Spinor Connection and Classical Approximation, arXiv:gr-qc/0610001

[13] Y. Q. Gu, New Approach to N-body Relativistic Quantum Mechanics, Int. J. Mod. Phys. A22:20072020(2007), arXiv:hep-th/0610153

[14] Jay D. Tasson, Lorentz violation, gravitomagnetism, and intrinsic spin, Phys. Rev. D 86, 124021 (2012)

[15] J. L. Said, J. Sultana, K. Z. Adami, Gravitomagnetic effects in conformal gravity, Phys. Rev. D 88, $087504(2013)$

[16] E. Hackmann, C. Lammerzahl, Generalized gravitomagnetic clock effect, Phys. Rev. D 90, 044059 (2014)

[17] E. Poisson, J. Doucot, Gravitomagnetic tidal currents in rotating neutron stars, Phys. Rev. D 95, 044023 (2017)

[18] B. Cashen, A. Aker, M. Kesden, Gravitomagnetic dynamical friction, Phys. Rev. D 95, 064014 (2017)

[19] Y. Q. Gu, Stationary Spiral Structure and Collective Motion of the Stars in a Spiral Galaxy, arXiv:0805.2828 